\title{
What can we learn from $(\mathrm{n}, x \mathrm{n} \gamma)$ cross sections about reaction mechanism and nuclear structure?
}

\author{
Maëlle Kerveno ${ }^{1, *}$, Marc Dupuis ${ }^{2}$, Catalin Borcea ${ }^{3},{\text { Marian } \text { Boromiza }^{3} \text {, Roberto Capote }}^{5}$, Philippe Dessagne ${ }^{1}$, Greg \\ Henning ${ }^{1}$, Stéphane Hilaire ${ }^{2}$, Toshihiko Kawano ${ }^{6}$, Alexandru Negret $^{3}$, Markus Nyman ${ }^{7}$, Adina Olacel ${ }^{3}$, Eliot Party ${ }^{1}$, \\ Arjan Plompen ${ }^{7}$, Pascal Romain ${ }^{2}$ and Mihaela $\mathrm{Sin}^{4}$ \\ ${ }^{1}$ Université de Strasbourg, CNRS, IPHC UMR 7178, Strasbourg, France \\ ${ }^{2}$ CEA, DAM, DIF, Arpajon, France \\ ${ }^{3}$ Horia Hulubei National Institute of Physics and Nuclear Engineering, Bucharest-Magurele, Romania \\ ${ }^{4}$ University of Bucharest, Faculty of Physics, Bucharest-Măgurele, Romania \\ ${ }^{5}$ IAEA, Nuclear Data Section, Vienna, Austria \\ ${ }^{6}$ Los Alamos National Laboratory, Los Alamos, New Mexico, 87545, USA \\ ${ }^{7}$ European Commission, Joint Research Centre, Geel, Belgium
}

\begin{abstract}
Inelastic (n,n') cross section is a key quantity to accurately simulate reactor cores, and its precision was shown to need significant improvements. To bypass the experimental difficulties to detect neutrons from $(\mathrm{n}, x \mathrm{n})$ reaction and to discriminate inelastically scattered neutrons from those following the fission process in case of fissile targets, an indirect but yet powerful method is used: the prompt $\gamma$-ray spectroscopy. Along this line, our collaboration has developed the GRAPhEME setup, optimized for actinides, at the GELINA facility to measure partial ( $\mathrm{n}, x \mathrm{n} \gamma)$ cross sections, from which the total $(\mathrm{n}, x \mathrm{n})$ cross section can be inferred. ( $\mathrm{n}, x \mathrm{n} \gamma)$ experiments with actinides are still particularly challenging, as their structure presents a high level density at low energy, and the competing neutron-induced fission reaction contaminates the $\gamma$-energy distribution. New precise measurements of the partial ( $\mathrm{n}, x \mathrm{n} \gamma$ ) cross sections provide a stringent test to theoretical model and offer a way to improve them. This is a path to a better determination of the total inelastic scattering cross sections. In this contribution we discuss modeling aspects of the ${ }^{238} \mathrm{U}$ and ${ }^{182} \mathrm{~W}\left(\mathrm{n}, \mathrm{n}^{\prime} \gamma\right)$ reactions, also measured with GRAPhEME, using the three codes TALYS, EMPIRE and CoH. We will highlight the needed/expected improvements on reaction modeling and nuclear structure input.
\end{abstract}

\section{Introduction}

Inelastic scattering reactions are important processes in a reactor core as they contribute to the slowing down of the neutrons and then have an impact on macroscopic core parameters as $k_{\text {eff }}$ or radial power distribution. The need for a better knowledge of their cross sections for fuel, coolant or structure materials has been highlighted since the nineties. In 1999, the NEA WPEC-subgroup 4 [1] has already emphasized the large discrepancies between the evaluated ${ }^{238} \mathrm{U}$ inelastic (and capture) cross sections and emphasized the need of new experimental and theoretical studies. This first report has motivated many experimental works. In 2008, the NEA WPEC-subgroup 26 has compiled, for several GEN-IV reactors, the cross sections accuracy needed to reach the target uncertainty for the reactor core parameters [2]. They concluded that reported uncertainty on the (n,n') cross section of about $20 \%$ is still too high to allow accurate simulations of core parameters. Several requests on (n,n') cross sections for various nuclei are listed in the HPRL [3]. Among other works, the recent studies performed by CEA/DEN [4] and PSI (Zürich) [5] have shown

*e-mail: maelle.kerveno@iphc.cnrs.fr the importance of good knowledge of the ${ }^{238} \mathrm{U}\left(\mathrm{n}, \mathrm{n}^{\prime}\right)$ cross section for the simulation of cores of commercial reactors. In this contribution, we report a joint experimental and theoretical effort to improve the quality of evaluated $(\mathrm{n}, \mathrm{n}$ ') cross sections. Section 2 describes the experimental work to produce new neutron inelastic scattering data with the prompt $\gamma$-ray spectroscopy method, while Section 3 shows, through specific examples with ${ }^{182} \mathrm{~W}$ and ${ }^{238} \mathrm{U}$ targets, how these data can be used to pinpoint modeling issues and to constraint model parameters.

\section{GRAPhEME and the prompt $\gamma$-ray spectroscopy method}

\subsection{The prompt $\gamma$-ray spectroscopy method for (n,n') studies}

Three experimental methods exist to measure (n, $x \mathrm{n})$ reaction cross sections. In this work, we use the prompt $\gamma$-ray spectroscopy combined with time of flight measurement to produce (n, $x \mathrm{n} \gamma$ ) cross sections [6]. This method gets rid of the difficulty to detect neutrons and can be used at white neutron beam facilities. The ( $\mathrm{n}, x \mathrm{n} \gamma$ ) cross sections 
Figure 1. (color online) Schematic view of the two ways to deduce total $(\mathrm{n}, x \mathrm{n})$ cross section from measured (n,n' $\gamma$ ) ones from ref. [7].

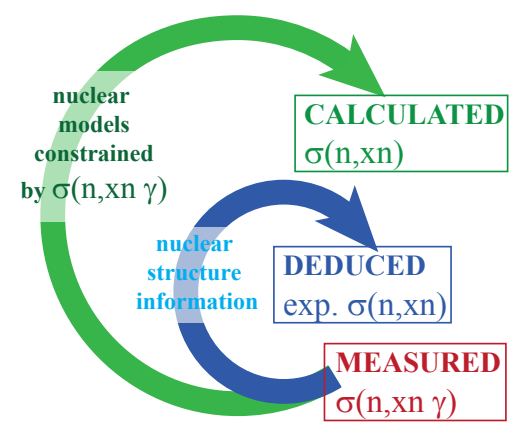

obtained are then used to infer the level production and total $(\mathrm{n}, \mathrm{xn})$ ones by two possible ways as illustrated in Fig. 1. This procedure has been explained in detail in Ref. [7].

We can already notice that nuclear structure knowledge is a key parameter for the inferring of inelastic cross section and in chapter 3 , we will show how the (n, $x \mathrm{n} \gamma$ ) cross sections can help to improve the modeling of different components of the inelastic process.

\subsection{GRAPhEME: characteristics and performances for $(\mathbf{n}, x \mathbf{n} \gamma)$ measurements}

Our experimental collaboration [IPHC (France), EC-JRCGeel (Belgium) and IFIN-HH (Romania)] has developed since almost fifteen years two germanium arrays for the study of neutron inelastic scattering at the GELINA (Geel Electron LINear Accelerator) neutron facility operated by EC-JRC-Geel [8]. The GAINS (Gamma Array for Inelastic Neutron Scattering) setup is mainly devoted to measurements on light and intermediate mass nuclei and is placed at a long flight path $(100 \mathrm{~m})$ (see contribution [9] in these proceedings). The second setup is called GRAPhEME (GeRmanium array for Actinides PrEcise MEasurements) and is placed at $30 \mathrm{~m}$ from the neutron source. It has been optimized for measurements on actinides and is composed of one fission chamber, which is used for neutron flux determination and, currently, of six planar germanium detectors including one segmented in 36 pixels. The typical neutron energy resolution is $10 \mathrm{keV}$ at $E_{n}=1 \mathrm{MeV}$. Measurements have been performed on ${ }^{232} \mathrm{Th}$ [10], ${ }^{233,235,238} \mathrm{U}$ [11], ${ }^{\text {nat }} \mathrm{Zr},{ }^{\text {nat }, 182,183,184,186} \mathrm{~W}$ [12] and ${ }^{57} \mathrm{Fe}$. The sample thickness ranged from 0.2 to $1.3 \mathrm{~mm}$ and the total beam time, for a measurement, varied between 400 hours and 3000 hours. GRAPhEME is then able to produce numerous ( $\mathrm{n}, x \mathrm{n} \gamma)$ cross sections $(x=1,2,3)$ with total uncertainties ranging from $3 \%$ to $20 \%$ depending on the incident neutron energy. A special effort is being made to publish well documented data regarding the analysis procedure and all uncertainty sources and their correlations explicitly described in view of an efficient use in the evaluation process.

\section{Modeling the $\left(n, n^{\prime} \gamma\right)$ reaction}

The production of a $\gamma$-ray (following the decay from a level $i$ to a level $j$ ) resulting from a neutron inelastic reaction is the product of two quantities: the production of

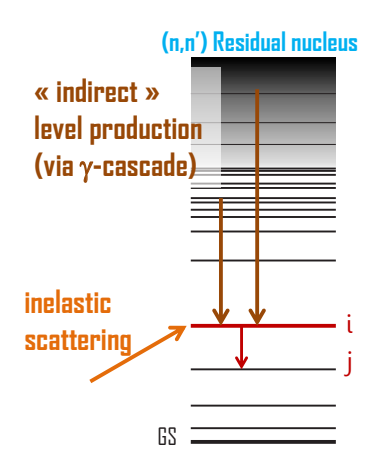

Figure 2. (color online) Schematic view of a residual nucleus after $(\mathrm{n}, \mathrm{n}$ ') reaction and decay by gammas of the produced level $i$.

the level $i$, and the probability $B R_{\gamma}^{i \rightarrow j}$ to decay to a daughter level $j$, that is the Branching Ratio (BR) (see Fig. 2).

We can distinguish two main components in the production of the level $i$. The first is the inelastic scattering of the neutron to the level $i$, with contributions from a direct $\sigma_{\text {Dir }}^{i}$, and a compound $\sigma_{\text {Comp }}^{i}$ (formation and decay of the compound nucleus) mechanisms. The second is the side feeding, namely the production of the level $i$ by the $\gamma$ cascade induced by the decay of levels produced at higher excitation energy. It splits into a "Discrete Cascade" part (decay from discrete levels) $\sigma_{\text {Dis. Casc. }}^{i}$ and a "Continuum Cascade" part (decay from continuum) $\sigma_{\text {Cont. Casc. Finally, }}^{i}$ the $\sigma\left(n, n^{\prime} \gamma_{i \rightarrow j}\right)$ cross section reads

$$
\begin{aligned}
\sigma\left(n, n^{\prime} \gamma_{i \rightarrow j}\right)=( & \sigma_{\text {Dir }}^{i}+\sigma_{\text {Comp }}^{i}+ \\
& \left.\sigma_{\text {Disc. Casc. }}^{i}+\sigma_{\text {Cont. Casc. }}^{i}\right) \times B R_{\gamma}^{i \rightarrow j}
\end{aligned}
$$

\subsection{What can we learn about reaction mechanisms?}

\subsection{1 (n,n' $\gamma)$ cross sections}

The comparison between experimental and calculated $(\mathrm{n}, \mathrm{n}$ ' $\gamma)$ cross sections may pinpoint model inaccuracies and thus help improving the whole modeling of the reaction. Nevertheless for specific transitions, differences between experimental data sets pose additional challenges. This is illustrated for two $\gamma$-rays in ${ }^{238} \mathrm{U}$ in Fig. 3, which compares the (n,n' $\gamma$ ) cross sections measured with GRAPhEME, and measurements from previous experiments, with three calculations performed with the codes TALYS [13] (model A), EMPIRE [14] (model B) and $\mathrm{CoH}[15]$ (model C). Note that i) model A corresponds to TALYS 1.8 where the pre-equilibrium model was replaced by the JLM/QRPA model [16]; ii) model B was used to produce the ${ }^{238} \mathrm{U}$ CIELO file, later adopted as ENDF/B-VIII.0. Figure 3 shows that, while the main features of the measured cross sections are qualitatively reproduced by the calculations, strong differences between the three calculations and the measurements are observed. A fully understanding of those discrepancies is a long and tedious process, but it is necessary to clarify which are the best choices of models, parameters and prescriptions to describe (n,n' $\gamma$ ) reactions, and eventually to better determine total inelastic (n,n') cross sections. By comparing 
calculations from models $\mathrm{A}, \mathrm{B}$ and $\mathrm{C}$ of the various components of Eq. 1, the present work reports first steps along this line.
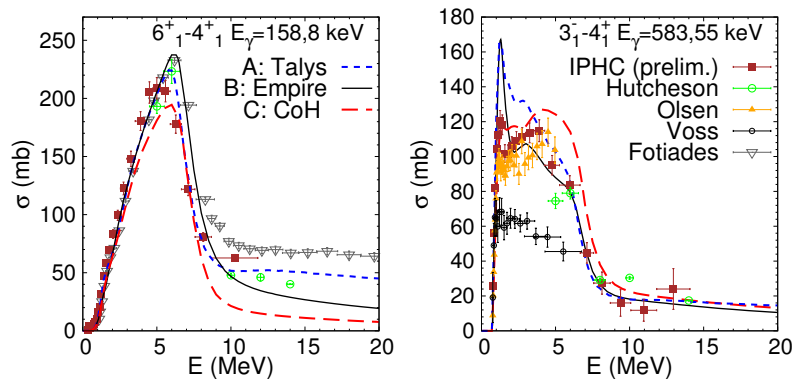

Figure 3. (color online) Experimental ${ }^{238} \mathrm{U}\left(\mathrm{n}, \mathrm{n}^{\prime} \gamma\right.$ ) cross sections (symbols defined in the right panel) for $\mathrm{E}_{\gamma}=158.8 \mathrm{keV}$ (Yrast transition, left panel) and for $\mathrm{E}_{\gamma}=583.55 \mathrm{keV}$ (inter band transition, right panel) compared with the nuclear reaction code calculations (defined on left panel).

\subsubsection{Level production cross sections}

We first review the various features involved in the calculations of the production of a specific level $i$. This production, obviously, strongly depends of model input parameters and prescriptions used to describe the various mechanisms at work. For inelastic scattering to the level $i$, these parameters are

(i) for the direct mechanism: optical potential parameters, coupling scheme (number of coupled states and angular momentum cut-off) and deformation lengths;

(ii) for the compound mechanism: optical potential parameters that provide the transmission coefficient, chosen method to account for widths fluctuations.

The determination of the side feeding depends on the probability to decay to the level $i$ from all the levels $k$ produced at a higher energy, and the production of each level $k$. It also depends on prescriptions chosen to describe the nuclear structure which is only partially known. Indeed, for actinides, the precise knowledge of the level scheme becomes incomplete at excitation energies as low as 11.2 MeV. Above this energy, the spectrum is described as a continuum, relying on specific choice of level densities. The number of discrete states, i.e. the energy at which the spectrum description switch from discrete to continuous, is an important parameter of the calculation. Note that the $\mathrm{CoH}$ code offers one specific possibility, that is to account for a discrete state in a continuum. Then, once the spectrum is defined, the production of the level $i$ after a $\gamma$ cascade from a level $k$ depends on

(iii) the $\gamma$-strength functions if $k$ belongs to the continuum,

(iv) the branching ratios, if $k$ is a discrete level,

(v) the production of level $k$; if $k$ is a discrete level, the elements of points i) and ii) are involved; if $k$ is in the continuum, its production involves elements of point ii) for a compound process, and the description of direct inelastic scattering to the continuum, namely the pre-equilibrium model.

Each of these modeling features will have different relative importance that depend on the level $i$ and the incident energy considered. This is illustrated in Fig. 4, which shows the productions of the $2_{1}^{+}$and $3_{1}^{-}$levels in ${ }^{238} \mathrm{U}$ from modeling $\mathrm{A}, \mathrm{B}$ and $\mathrm{C}$. The total level production (dashed curves), and its inelastic scattering component (solid curves) are displayed. The differences in shape between model A, B and C can be related to specific aspects of the model.
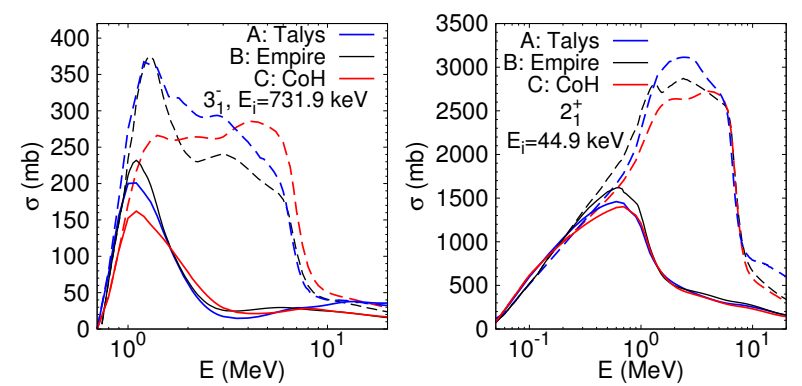

Figure 4. (color online) level production cross sections (dashed curves) in ${ }^{238} \mathrm{U}$ with inelastic component (full curves) for $\mathrm{E}_{i}=44.9 \mathrm{keV}$ (Ground state rotational band, right panel) and for $\mathrm{E}_{i}=731.9 \mathrm{keV}$ (rotational band 2, left panel) from models A, B and $\mathrm{C}$.

\subsection{What can we learn about nuclear structure?}

\subsubsection{Continuum cascade cross sections}

Features of the modeling of the side-feeding from continuum states can be examined from specific (n,n' $\gamma$ ) transitions. D. Dashdorj el al. [17] showed that a microscopic description of the population of continuum states via direct and pre-equilibrium process lead to a better description of some (n,n' $\gamma$ ) reactions. This was related to the spin distribution associated to the residual nucleus which can be predicted by the microscopic pre-equilibrium model. In Ref. [18], a similar approach was successfully applied to the ${ }^{238} \mathrm{U}(\mathrm{n}, \mathrm{n}$ ' $\gamma$ ) reaction for transitions from high spin states belonging to the ground state rotational band (GSRB). We illustrate in Fig. 5 this feature by comparing, for two transitions in ${ }^{238} \mathrm{U}$, measurements and calculations from models A, B and C. Models A and B used a microscopic description of pre-equilibrium emission (respectively the folding JLM/QRPA model of Ref. [18] and the multistep direct model of Ref. [19]), while model C relies on the exciton model with $n$-exciton spin distributions [14].

Other ingredients of the continuum cascade modeling that we can test are the $\gamma$-strength functions. Similar to the pre-equilibrium model, we have observed that a more microscopic description (using QRPA nuclear structure calculations) of the E1 and M1 has a huge impact on inter- 

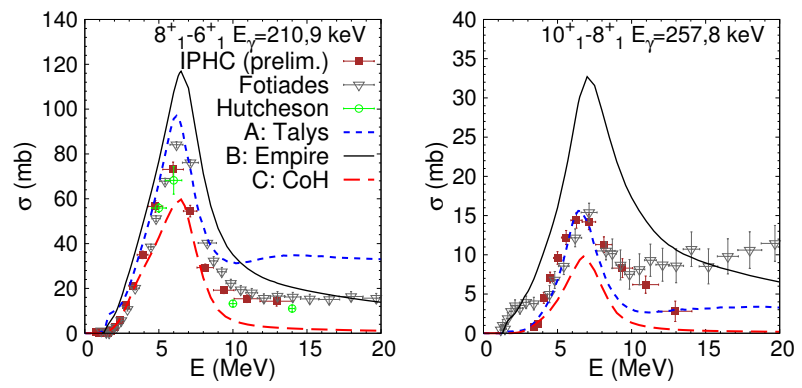

Figure 5. (color online) Experimental ${ }^{238} \mathrm{U}\left(\mathrm{n}, \mathrm{n}^{\prime} \gamma\right.$ ) cross sections for $\mathrm{E}_{\gamma}=210.9 \mathrm{keV}$ (left panel) and for $\mathrm{E}_{\gamma}=257.8 \mathrm{keV}$ (right panel) compared to the nuclear reaction code calculations.

band $\gamma$ transitions and leads to better cross section description [20].

\subsubsection{Discrete cascade cross sections}

While in ${ }^{238} \mathrm{U}$, microscopic pre-equilibrium models lead to a realistic account of high spin $\gamma$ transitions in the GSRB (see section 3.2), this is not the case for ${ }^{182} \mathrm{~W}$, as shown in Fig. 6 by continuous blue curves (model A) and dashed red curves (model C). A recent update of model C, which now accounts for 70 discrete levels up to $2.3 \mathrm{MeV}$ (compared to 29 levels up to $1.8 \mathrm{MeV}$ in the previous model $\mathrm{C}$, and similar prescriptions in model A and B) and embeds discrete levels in the continuum, seems to solve this issue as shown by the red continuous line in Fig. 6 .
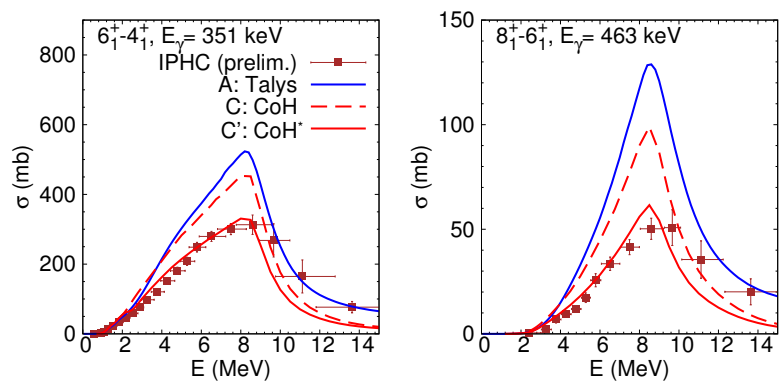

Figure 6. (color online) Experimental ${ }^{182} \mathrm{~W}\left(\mathrm{n}, \mathrm{n}^{\prime} \gamma\right)$ cross sections for intra-band transitions (left panel: $\mathrm{E}_{\gamma}=351 \mathrm{keV}$, right panel: $\mathrm{E}_{\gamma}=463 \mathrm{keV}$ ) compared to the nuclear reaction code calculations.

\subsubsection{Nuclear structure aspects}

Some levels branching ratios between different deexcitation paths can be measured with GRAPhEME and compared with literature values ( ENSDF [21]). Scanning over the 26 analyzed levels in ${ }^{232} \mathrm{Th}$, we have found some differences for transitions from three levels (at 873, 1042.9 and $1121.7 \mathrm{keV}$ ). For ${ }^{238} \mathrm{U}$, the analysis is still in progress but a first significant difference has been found for transitions from the $3_{1}^{-}$level at $680.11 \mathrm{keV}$.

The method we used to infer the (n,n') cross section is strongly dependent of the nuclear structure knowledge. It is thus a huge concern for our team to quantify the sensitivity of variation in the description of the level scheme. For example in ${ }^{238} \mathrm{U}$ and up to $\mathrm{E}_{i}=1.3 \mathrm{MeV}$ (the maximum excitation energy reached in our experiment), $79 \gamma$ transitions are listed in ENSDF, 76\% have BR information and only $56 \%$ are mentioned with uncertainty. To help quantifying the consequence of this, we have developed a Monte Carlo simulation based on TALYS 1.8 code which allows to estimate the sensitivity of a $\gamma$ transition cross section to the uncertainty on BRs of other $\gamma$ transitions. Figure 7 shows the sensitivity matrix obtained for ${ }^{238} \mathrm{U}$. The main conclusions are that, up to excitation energies around $780 \mathrm{keV}, \gamma$-production cross sections are sensitive to the BRs and an uncertainty on BR of $10 \%$ leads to an uncertainty on cross section of around $4 \%$. In ENSDF, the average BR uncertainty is $8 \%$, thus this effect has to be considered when inferring the (n,n') cross section from $(\mathrm{n}, \mathrm{n}$ ' $\gamma)$ ones.

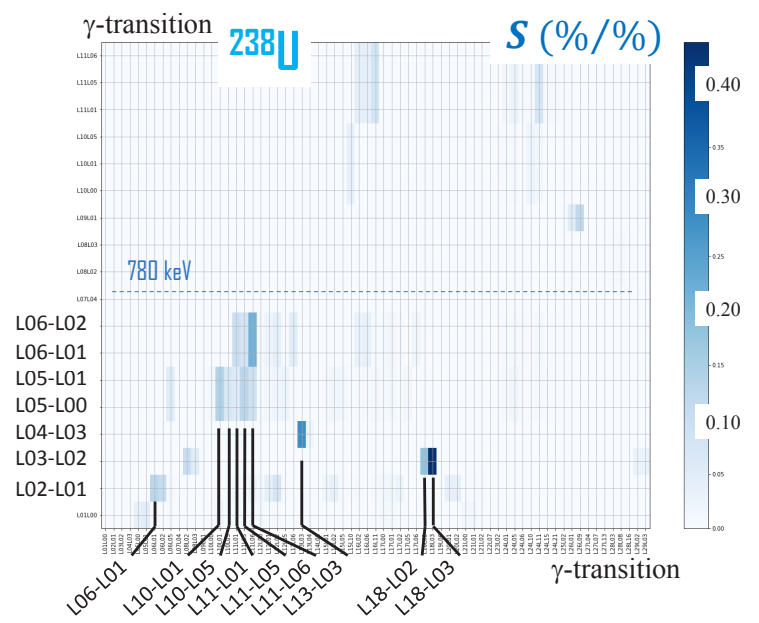

Figure 7. (color online) Sensitivity matrix for $\gamma$-production cross sections to Branching Ratios in ${ }^{238} \mathrm{U}_{.} \mathrm{L}_{i}$ is the number of the level in TALYS.

\section{Conclusions}

The prompt $\gamma$-ray spectroscopy method with the GRAPhEME setup is a powerful method to produce precise $(\mathrm{n}, x \mathrm{n} \gamma)$ cross sections for valuable evaluation. In this work, we have shown that the (n,n' $\gamma$ ) cross sections can be used as a fine probe to pinpoint shortcomings in both reaction modeling and nuclear structure knowledge. This can be used to improve the models. Results reported here are only the first step of a more comprehensive work which will lead to well documented publications for ${ }^{238} \mathrm{U}$, ${ }^{182,184,186} \mathrm{~W}$ and ${ }^{232} \mathrm{Th}$

The authors thank the GELINA and IPHC staff for their support. This work was partially supported by NEEDS, PACEN/GEDEPEON, French ANR and by the European Commission within the Sixth Framework Program through I3EFNUDAT (EURATOM contract Nr. 036434) and NUDAME (Contract FP6-516487), and within the Seventh Framework Program through EUFRAT (EURATOM contract Nr. FP7-211499), 
through ANDES (EURATOM contract Nr. FP7-249671) and through CHANDA (EURATOM contract Nr. FP7-605203).

\section{References}

[1] Y. Kanda and M. Baba, Nuclear ScienceNEA/WPEC-4, vol. 4, (1999).

[2] M. Salvatores and R. Jacqmin, Nuclear ScienceNEA/WPEC-26, Volume 26, NEA N 6410 (2008).

[3] OECD/NEA, Nuclear Data High Priority Request List, http://www.nea.fr/dbdata/hprl/.

[4] A. Santamarina et al. Nucl. Data Sheets 118, 118-121 (2014).

[5] D. Rochman et al. Ann. Nucl. Energy. 112, 236-244 (2018)

[6] M. Kerveno et al. Eur. Phys. J. A 51, 167, (2015).

[7] M. Kerveno et al. Eur. Phys. J. N 4, 23, (2018).

[8] D. Ene et al. Nucl. Instrum. Methods Phys. Res. A 618, 54 (2010).

[9] A. Negret et al., Proceedings of the ND2019 conference, Beijing, China (2019).

[10] E. Party et al. Eur. Phys. J Web of Conferences 211, 03005, (2019)
[11] M. Kerveno et al. Phys. Rev. C 87, 24609 (2013).

[12] G. Henning et al. Eur. Phys. J. Web of Conference 146, 11016, (2017)

[13] A.J. Koning et al. Proc. of the Int. Conf. on Nucl. Data for Science and Technology - ND2004, AIP vol. 769, eds. R.C. Haight, M.B. Chadwick, T. Kawano, and P. Talou, Sep. 26 - Oct. 1, 2004, Santa Fe, USA, p. 1154 (2005).

[14] M. Herman et al., internal report from Brookhaven National Laboratory, INDC(NDS)-0603, BNL101378-2013 (2013).

[15] T. Kawano, Proc. of the Int. Workshop on Coumpound Nuclear Reactions and Related Topics, Berkeley (2018), to be published.

[16] M. Dupuis, et al., Eur. Phys. J. A 51, 168, (2015).

[17] D. Dashdorj el al., Phys. Rev. C 75, 054612 (2007).

[18] M. Dupuis et al., Eur. Phys. J. Web of Conference 146, 12002, (2017)

[19] T. Kawano et al., Phys. Rev. C 63, 034601 (2001).

[20] S. Hilaire et al., Proceedings of the ND2019 conference, Beijing, China (2019).

[21] P. Dimitriou et al., Proceedings of the ND2019 conference, Beijing, China (2019). 